2006 [1]. Intellectual disability (ID) is characterized by significant limitations both in intellectual functioning and in adaptive behavior as expressed in conceptual, social and practical adaptive skills. The disability originates before age 18 years, and the prevalence is estimated at $1 \%$ to $3 \%$. Global development delay (GDD) is defined as a significant delay in 2 or more developmental domains, including gross or fine motor, speech/language, cognitive, social/personal, and activities of daily living, and is thought to predict a future diagnosis of ID. GDD is applied to younger children $<5$ years, and the prevalence is $1 \%$ to $3 \%$, similar to that of ID. The term ID is applied to older children for whom IQ testing is valid and reliable.

In patients with GDD or ID, chromosome microarray and fragile $\mathrm{X}$ are first line diagnostic tests. MRI remains important in some patients. The use of whole-exome sequencing as a diagnostic test is gaining popularity. (Moeschler JB, Shevell M. Committee on Genetics. Comprehensive evaluation of the child with intellectual disability or global development delays. Pediatrics 2014 Sep;134(3):e903-18).

COMMENTARY. The benefits of a comprehensive evaluation were studied in 20 families of children with ID, with and without an etiologic diagnosis [2]. A diagnosis was thought to help guide expectations and management and provide hope for treatment. Diagnosis assisted families in obtaining desired services, especially in schools. Families differed in their "need to know" a specific diagnosis, ranging from strong to indifferent. Families varied in their emotions and actions regarding prenatal genetic diagnosis. The importance of the pediatrician and clinical geneticist discussion is stressed before deciding on the best approach to the diagnostic evaluation. The pediatric neurologist has an equally important role in the arrival at the correct etiological diagnosis and management of a child with GDD or ID.

\title{
References.
}

1. Moeschler JB, Shdevell M. Pediatrics. 2006 Jun;117(6):2304-16.

2. Makela M, et al. Am J Med Genet A. 2009 Nov;149A(11):2393-402.

\section{SUBDURAL HEMORRHAGE AND ANTITHROMBOTIC THERAPY}

Investigators at the Mott Children's Hospital, Ann Arbor, MI, report 4 infants with diffuse brain injury and cerebral atrophy who developed cerebral venous sinus thrombosis and were treated with the low-molecular-weight heparin, enoxaparin. The infants subsequently developed subdural hemorrhages, diagnosed on routine MRI, and one had focal seizures. The use of enoxaparin in infants with diffuse brain injury has a risk of intracranial subdural hemorrhage. (Dang LT, Shavit JA, Singh RK, et al. Subdural hemorrhages associated with antithrombotic therapy in infants with cerebral atrophy. Pediatrics 2014 Sep;134(3):e889-93).

COMMENTARY. Asymptomatic subdural hematomas are common in infants with congenital heart disease and they resolve within 3 months of birth [1].

References.

1. Kelly P, et al. Pediatrics. 2014 Sep;134(3):e773-81. 